\title{
MRI but not arthroscopy accurately diagnoses femoral MPFL injury in first-time patellar dislocations
}

\author{
Peter Balcarek • Tim Alexander Walde • \\ Stephan Frosch • Jan Philipp Schüttrumpf • \\ Martin Michael Wachowski · Klaus Michael Stürmer
}

Received: 7 June 2011/Accepted: 8 November 2011/Published online: 18 November 2011

(C) The Author(s) 2011. This article is published with open access at Springerlink.com

\begin{abstract}
Purpose The purpose of this study was to investigate whether the femoral part of the medial patellofemoral ligament (MPFL) and its injury can be accurately assessed by standard knee arthroscopy in first-time patellar dislocations or whether preoperative MRI is required to determine injury location in patients where primary MPFL repair is attempted.

Methods Twelve patients with acute first-time dislocations and MRI-based injury of the femoral MPFL and ten patients with recurrent patellar dislocations underwent knee arthroscopy with the use of a 30-degree optic and standard antero-medial and antero-lateral portals. The femoral origin was marked with a cannula under lateral fluoroscopy. Arthroscopic findings of the location of the native femoral MPFL and its injury were compared to the results of MRI and mini-open exploration.

Results In acute cases, the average time from primary patellar dislocation to MRI evaluation was 3 days (1-9 days), and the average time from MRI to surgery was 8 days (3-20 days). The native femoral origin of the MPFL was not visible in any of the chronic cases during arthroscopy. In addition, in all acute cases, arthroscopy failed to directly visualize injury of the femoral MPFL ( 0 of 12), but mini-open exploration confirmed injury in 11 of 12 patients. This means that arthroscopy was less accurate than MRI for the diagnosis of femoral MPFL injury $(P<0.05)$.
\end{abstract}

P. Balcarek $(\bowtie) \cdot$ T. A. Walde $\cdot$ S. Frosch .

J. P. Schüttrumpf · M. M. Wachowski - K. M. Stürmer

Department of Trauma Surgery, Plastic and Reconstructive

Surgery, University Medicine, Robert-Koch-Str. 40,

37075 Göttingen, Germany

e-mail: peter.balcarek@med.uni-goettingen.de
Conclusion The results of this study indicate the limitations of knee arthroscopy in identifying the femoral disruption of the MPFL, a crucial injury that occurs in patellar dislocations. Thus, if a primary MPFL repair is planned, determination of the site of repair should be based on the preoperative MRI.

Level of evidence Diagnostic study of non-consecutive patients, Level III.

Keywords MPFL · Patellar dislocation - Arthroscopy · MRI

\section{Introduction}

The treatment of lateral patellar dislocation (LPD) is subject to constant advancement and requires a profound understanding of the complex interplay of individual predisposing factors and injury patterns. Though most authors favour a conservative therapeutic regime after a first-time dislocation, several circumstances may warrant surgical intervention. These circumstances include an osteochondral fragment amendable to refixation, relevant cartilage damage or a relevant disruption of the medial ligamentous stabilizers with subluxation of the patella and normal contralateral patellar tracking [17]. In this context, the medial patellofemoral ligament (MPFL) has attained increased significance in recent years. This ligament represents the major component of the medial ligamentous complex and maintains the stability of the patellofemoral joint [7]. Injury to this ligament reduces passive stability by about $50-60 \%$ and may predict subsequent instability after conservative treatment [16]. Therefore, several authors have advocated the role of MPFL repair in the treatment of acute LPD and have described the results of performing 
MPFL reinsertion at the femoral origin or at the patellar attachment site with good results in terms of redislocation $[1,4]$.

The MPFL is a distinct structure in the second layer of the medial retinacular complex just superficial to the joint capsule [18]. It originates from the adductor tubercle, medial epicondyle and superficial MCL and inserts on the upper two-thirds of the medial patellar border. Magnetic resonance imaging (MRI) has been proven to be a reliable test for the detection of the MPFL injury pattern with a sensitivity and accuracy of 85 and $80 \%$, respectively, when compared to open exploration $[11,13]$.

There is no conclusive evidence that primary MPFL repair in first-time patellar dislocations reduces the incidence of recurrence $[1,4,5,10]$. However, if a primary MPFL repair is attempted, determination of the site of injury is crucial to achieve a satisfactory result. Although it would be logical to assume that injury to the MPFL, particularly to its femoral origin, being an extra-articular ligament is not amendable to arthroscopic evaluation, there is no evidence in the literature to this effect. Thus, the purpose of the current study was to investigate whether the femoral part of the MPFL and its injury can be accurately assessed by standard knee arthroscopy in first-time patellar dislocations or whether preoperative MRI is required to determine injury location in patients where primary MPFL repair is attempted. It was hypothesized that, as a result of the extrasynovial location of the MPFL, neither the native femoral origin nor its injury at the femoral attachment site is visible during standard knee arthroscopy.

\section{Materials and methods}

The institutional review board of the University Medicine Göttingen (Ref. Nr. 6/2/11) approved this study. Between January 2010 and March 2011, 53 patients were treated with lateral patellar instability. Twenty-five patients had first-time dislocations, and 28 patients had recurrent dislocations. Ten patients with recurrent patellar dislocations underwent knee arthroscopy and MPFL augmentation. These patients were evaluated to determine whether the femoral origin of the MPFL is visible during standard knee arthroscopy. Therefore, the femoral origin was marked with a cannula under lateral fluoroscopy (Fig. 1). The origin was determined according to the method described by Schoettle et al. [14]. After placement of the cannula, it was determined whether the cannula could be identified arthroscopically with the use of a 30-degree optic and standard antero-medial and antero-lateral portals. Thereafter, patients underwent MPFL augmentation with the use of an autologous gracilis tendon. Verifying femoral tunnel placement radiographically is recommended [15]; thus, the

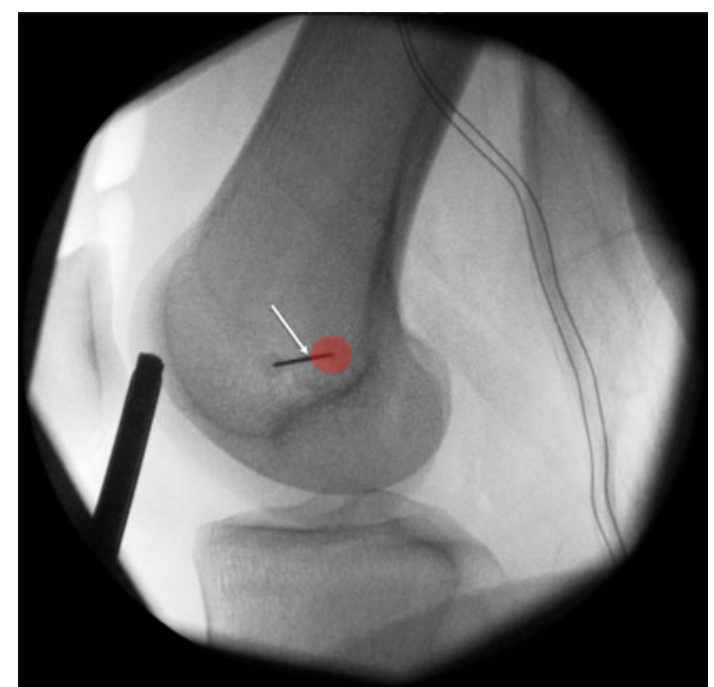

Fig. 1 The femoral origin of the MPFL (red circle) was determined under lateral fluoroscopy according to the method described by Schoettle et al. [14] and was marked with a cannula (arrow)

cannula was used to guide anatomical femoral tunnel positioning.

To test whether injury of the MPFL at the femoral origin can be accurately diagnosed by arthroscopy, 12 patients with acute first-time dislocations (12 of 25) and proven MRI-based injuries of the femoral MPFL (please see below) underwent standard knee arthroscopy. The indicator for primary surgery after first-time dislocation was an osteochondral fragment and/or a complete disruption of the medial ligamentous stabilizers [17]. Again, standard knee arthroscopy was performed with the use of a 30-degree optic and antero-medial and antero-lateral portals. Two experienced surgeons assessed the location of the injury with agreement by consensus. After arthroscopic assessment, a third surgeon provided the findings of the previously performed MRI. Based on both the MRI findings and the arthroscopic evaluation, patients underwent further surgery that included refixation of the torn MPFL at the femoral origin, direct suture of the injured ligamentous structures and/or refixation or removal of osteochondral fragments or loose bodies. For MPFL refixation or MPFL suture, a mini-open exploration was performed.

\section{MRI technique and image evaluation}

Prior to surgery, magnetic resonance imaging of the knee was performed in all patients. All magnetic resonance (MR) examinations were performed on a 1.5- or 3.0-T imager (Magnetom TrioTim Syngo MR B15 and Magnetom Symphony Syngo MR A30, Siemens, Erlangen, Germany). Patients underwent imaging with the knee positioned in full extension. A transverse, fat-saturated, proton 
density-weighted fast-spin-echo imaging sequence was performed routinely and was used for the evaluation of MPFL injury patterns according to Elias et al. [8]. The MRI results were evaluated by two orthopaedic surgeons with agreement reached by consensus. The MPFL was visualized as the lowsignal intensity fibres originating between the adductor tubercle and the medial epicondyle running inferior to the vastus medialis obliquus (VMO) and inserting at the proximal two-thirds of the medial patellar facet. The MPFL structure was divided into three regions: femoral origin, midsubstance and patellar insertion. The fibres of the MPFL were assessed to be normal, partially disrupted or completely disrupted. A partial disruption was expected when some of the fibres were identified, but a partial discontinuity or irregularity with intraligamentous or periligamentous oedema was obvious. A complete disruption was expected when a complete discontinuity of the fibres with extended surrounding oedema was obvious. MRI-based classification of MPFL injury patterns was performed according to Balcarek et al. (Table 1) [2].

\section{Statistical analysis}

Values are presented as median and range. To compare the accuracy of MRI and arthroscopy as a diagnostic tool with regard to femoral MPFL injury, the true-positive rate of MRI and arthroscopy was calculated by Fisher's exact test. Statistical analyses were performed with GraphPad Prism (Prism 4 Statistics Guide Statistical analyses for laboratory and clinical researchers, GraphPad Software, Inc., San Diego, California, USA, 2003). A P value less than 0.05 was considered statistically significant.

\section{Results}

Patients with recurrent patellar dislocations consisted of five men and five women with a median age of 23 years (17-35 years). Patients had a median of 3 patellar dislocations (2-5) prior to surgery. Using the arthroscopic settings used in this study, the cannula was not directly visible in any patient. This means that it was not possible to assess the native femoral origin of the MPFL with standard knee arthroscopy.

Patients with a first-time dislocation included seven female and five male patients with a median age of

Table 1 Classification of MPFL injury patterns according to Balcarek et al. [2]

\begin{tabular}{ll}
\hline Type I & MPFL tear at the patellar insertion \\
Type II & MPFL midsubstance injury \\
Type III & MPFL tear at the femoral origin \\
Type IV & Combined injury \\
\hline
\end{tabular}

18 years (13-42 years). The average time from dislocation to MRI evaluation was 3 days (1-9 days), and the average time from MRI to surgery was 8 days (3-20 days). MRI investigations showed injury of the MPFL at the femoral origin in seven patients (Type III lesion) and combined lesions at the femoral origin and at the patellar insertion site (Type IV lesion) in five patients (Figs. 2, 3). Injuries were classified as complete in 5 cases and partial in 7 cases. Osteochondral flake fractures were found in 7 patients.

Arthroscopy failed to show direct injury of the femoral MPFL in all patients with Type III and Type IV lesions ( 0 of 12). However, haemorrhages shone through the synovial tissue in 3 patients, and these can be assessed as indirect signs of femoral MPFL injury (Fig. 4). However, only after transection of the synovial membrane, the haematoma became obvious during arthroscopy and could be confirmed as injury of the MPFL by mini-open exploration in 11 of 12 patients (Fig. 5a-c). This means that arthroscopy was less accurate than MRI for the diagnosis of femoral MPFL injury $(P<0.05)$. In addition, MRI and arthroscopic appearance indicated injury at the patellar insertion in patients with a Type IV injury (Fig. 6a, b). However, evaluation of the medial patellar margin using a palpation hook with concomitant miniopen exploration could not confirm a substantial disruption of the medial ligamentous complex at the patellar insertion.

\section{Discussion}

The most important finding of this study was that the femoral disruption of the MPFL, even in the acute setting of a first-time patellar dislocation, might not be reliable diagnosed by standard knee arthroscopy. Solely,

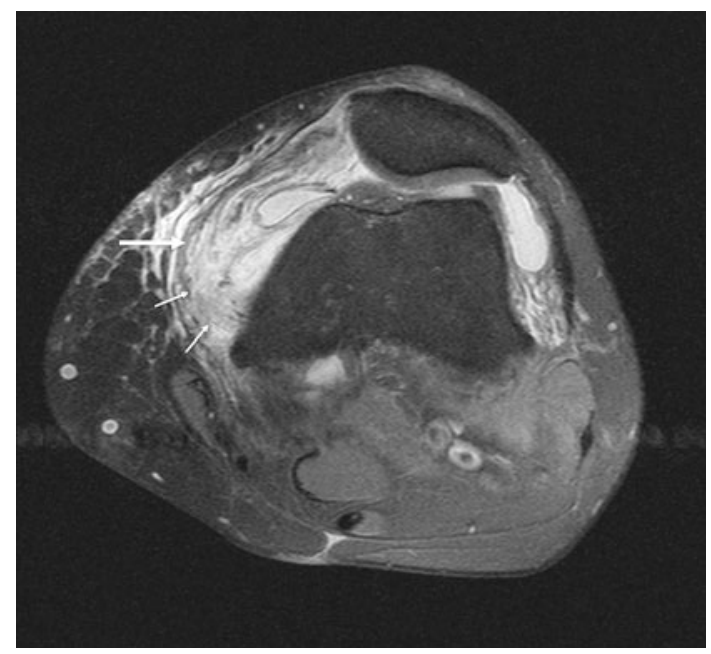

Fig. 2 The appearance of a complete femoral MPFL injury with retraction of the MPFL fibres anteriorly (thick arrow) and surrounding oedema (small arrows) in a 25-year-old woman 5 days after primary patellar dislocation 


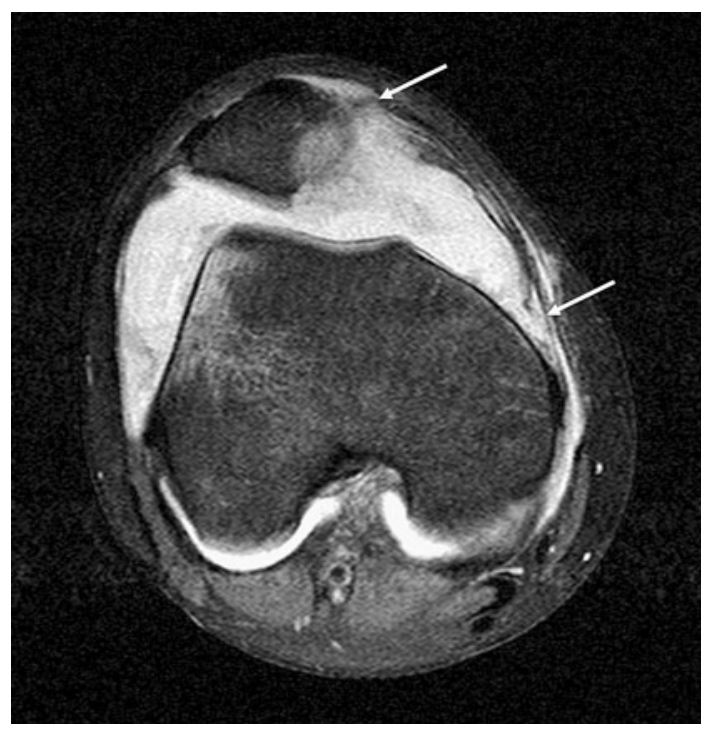

Fig. 3 The appearance of a combined injury of the MPFL with tear, surrounding oedema near the adductor tubercle and discontinuity at the patellar insertion (arrows) in a 14-year-old girl 2 days after primary patellar dislocation

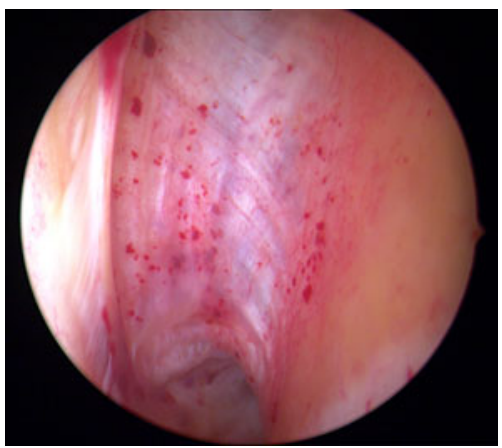

Fig. 4 Arthroscopic appearance of the medial recessus in a 25-yearold woman with an MRI-based complete disruption of the MPFL (please see Fig. 2) 20 days after patellar dislocation. The synovial tissue is intact with some blood residues and haematoma shining through the synovial membrane. The MPFL injury is not directly visible

haemorrhages that shone through the synovial tissue in projections of the femoral MPFL in several patients can be assessed as indirect signs of femoral MPFL injury. In addition, open exploration could not confirm a substantial disruption of the medial ligamentous complex at the patellar insertion in patients with MRI-based injuries at both the femoral origin and the patellar insertion of the MPFL.

The MPFL has been identified as the primary ligamentous restraint, accounting for $50-60 \%$ of the total restraining force against LPD [7]. Its injury occurred in over $90 \%$ of LPDs and might predict a subsequent instability after conservative treatment [16]. Therefore, several authors have advocated the role of MPFL repair in the
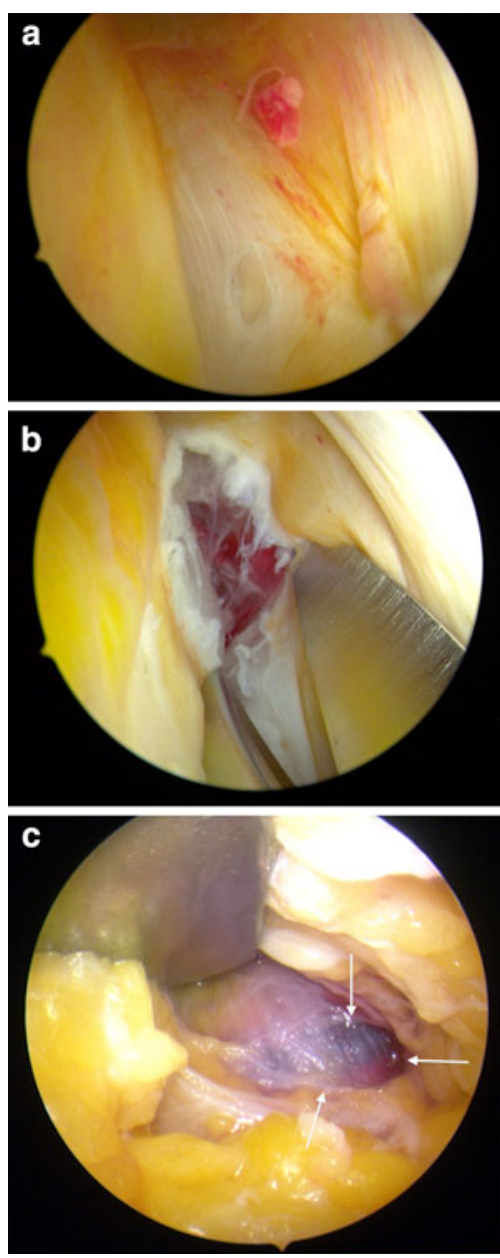

Fig. 5 Arthroscopic appearance of the medial recessus in a 13-yearold girl 9 days after primary patellar dislocation (a). After transection of the synovial membrane, haematomas became obvious in projections of the femoral MPFL (b), and partial tearing and stretching of the MPFL (arrows) were confirmed by mini-open exploration (c)

treatment of acute LPD $[1,4,10]$. However, injury patterns of the MPFL vary in cases of LPD. Tears have been found at the medial patellar margin, in the midsubstance and at the femoral origin [8]. In addition, MRI studies found more than one site of injury, most frequently located at the femoral origin and the patellar insertion sites [2, 8]. It has recently been shown that these injury patterns may vary in accordance with the magnitude of trochlear dysplasia, patella alta and the tibial tuberosity-trochlear groove (TT-TG) distance [2]. However, the problem of defining the exact location of an MPFL tear after a primary episode of dislocation remains. There is no agreement on this issue, but it has a clear surgical implication since errors in the interpretation of the MPFL injury location can compromise a successful surgery [10].

Based on open exploration, several authors advocate that injury of the MPFL occurs almost invariably at or close to its femoral attachment site $[11,13]$. At the time of 

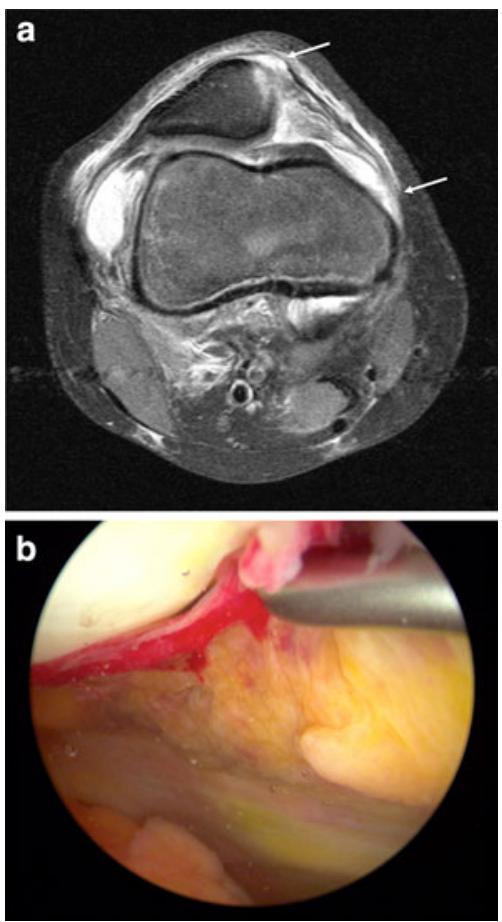

Fig. 6 MRI shows combined injury of the MPFL with tear, surrounding oedema near the adductor tubercle and discontinuity at the patellar insertion (arrows) in a 13-year-old girl 3 days after primary patellar dislocation (a). Arthroscopy discovered haematoma at the patellar margin (b), but mini-open exploration could not confirm a substantial disruption of the medial ligamentous complex

surgical exploration, Sanders et al. [13] found some degree of MPFL injury in all of their fourteen patients after dislocation. The MPFL was either completely disrupted near the femoral attachment site or demonstrated stretching or partial tearing. The MPFL was thought to be intact adjacent to the patellar insertion in all fourteen cases. Salley et al. [12] found tears of the femoral insertion of the MPFL in 15 of 16 cases, and surgical exploration confirmed injury of the MPFL at the femur in the same number of cases. In addition, Nomura et al. [11] characterized two types of MPFL injuries following acute patellar dislocation, the avulsion-tear type and the substantial tear type. Both types of injury were located near the femoral origin, superoposterior to the medial femoral epicondyle and just distal to the adductor tubercle.

Though Mariani et al. [10] most recently reported on a series of MPFL avulsions located at the patellar margin that were detected by MRI and confirmed by arthroscopy, the accuracy of arthroscopy in detecting femoral MPFL injuries has not been defined in the current literature. Thus, this study aims to provide a critical aspect on the diagnosis of MPFL injuries located at the femoral origin. It was hypothesized that, as a result of the extrasynovial location of the MPFL, its injury at the femoral attachment site is not accessible during arthroscopy. The hypothesis was proven correct since the results of this study indicate that even acute femoral injuries of the MPFL were not directly visible during arthroscopy. In a few patients (3 of 12), haemorrhages shone through the synovial tissue in projections of the femoral MPFL; these observations can be assessed as indirect signs of femoral MPFL injuries. However, without transection of the synovial tissue, the detection of injury would have been completely missed in 11 of 12 patients (in one patient, open exploration could not confirm femoral MPFL injury as shown by MRI). This is meaningful because femoral avulsions of the MPFL predict subsequent instability episodes after non-operative treatment [16]. In addition, the question has recently been raised as to whether a Type IV injury really comprises a substantial tear at two sites of the MPFL [3]. In this series, open exploration could not confirm a substantial disruption of the MPFL near the patellar insertion in cases of Type IV injury patterns but could detect femoral injury. These findings are in agreement with the results presented by Sanders et al. [13]. In their study, MRI was able to demonstrate disruption of the MPFL near the patella in a single case; however, this was not confirmed surgically.

By the 1990s, the literature suggested that patients with disruption of the MPFL might benefit from primary ligament repair following primary patellar dislocations $[9,12]$. Although not agreed upon, recent studies from the orthopaedic community have revived these findings [4, 10]. However, delayed primary repair of the MPFL did not reduce the risk of redislocation when compared to conservative treatment after a mean of 2-year follow-up [5]. Thus, successful surgery requires both an understanding of injury patterns and injury location and a detailed analysis of the individual factors that lead to predisposition to LPD [6].

This study indicates limitations of knee arthroscopy in identifying the femoral disruption of the MPFL in patellar dislocations; concurrently, it emphasizes the importance of a preoperative MRI investigation for a correct assessment of injury patterns and injury location in patients where primary MPFL repair is attempted. However, several limitations were noticed and deserve mentioned. First, this study included only a limited number of patients. This fact resulted from very stringent inclusion criteria, that is, a selected group of acute first-time dislocations with concomitant indications for primary surgery, and MRI and arthroscopic evaluation of the soft-tissue restraints as close as possible to the dislocating event. Second, statistical analysis was limited with regard to the accuracy of MRI and arthroscopy in detecting the true-positive rate of femoral MPFL injuries. The fact that only patients with MRI-based injury of the MPFL were included made it impossible to test for sensitivity and specificity. 


\section{Conclusion}

The results of this study indicate the limitations of knee arthroscopy in identifying the femoral disruption of the MPFL, a crucial injury that occurs in patellar dislocations. Thus, if a primary MPFL repair is planned, determination of the site of repair should be based on the preoperative MRI.

Conflict of interest The authors declare that they have no competing interests.

Open Access This article is distributed under the terms of the Creative Commons Attribution Noncommercial License which permits any noncommercial use, distribution, and reproduction in any medium, provided the original author(s) and source are credited.

\section{References}

1. Ahmad CS, Stein BE, Matuz D, Henry JH (2000) Immediate surgical repair of the medial patellar stabilizers for acute patellar dislocation. A review of eight cases. Am J Sports Med 28:804-810

2. Balcarek P, Ammon J, Frosch S, Walde TA, Schüttrumpf JP, Ferlemann KG, Lill H, Stürmer KM, Frosch K-H (2010) Magnetic resonance imaging characteristics of the medial patellofemoral ligament lesion in acute lateral patellar dislocations considering trochlear dysplasia, patella alta, and tibial tuberositytrochlear groove distance. Arthroscopy 26:926-935

3. Balcarek P, Walde TA, Frosch S, Schüttrumpf JP, Wachowski MM, Stürmer KM, Frosch KH (2011) Patellar dislocations in children, adolescents and adults: a comparative MRI study of medial patellofemoral ligament injury patterns and trochlear groove anatomy. Eur J Radiol 79:415-420

4. Camanho GL, Viegas Ade C, Bitar AC, Demange MK, Hernandez AJ (2009) Conservative versus surgical treatment for repair of the medial patellofemoral ligament in acute dislocations of the patella. Arthroscopy 25:620-625

5. Christiansen SE, Jakobsen BW, Lund B, Lind M (2008) Isolated repair of the medial patellofemoral ligament in primary dislocation of the patella: a prospective randomized study. Arthroscopy 24:881-887
6. Dejour H, Walch G, Nove-Josserand L, Guier C (1994) Factors of patellar instability: an anatomic radiographic study. Knee Surg Sports Traumatol Arthrosc 2:19-26

7. Desio SM, Burks RT, Bachus KN (1998) Soft tissue restraints to lateral patellar translation in the human knee. Am J Sports Med 26:59-65

8. Elias DA, White LM, Fithian DC (2002) Acute lateral patellar dislocation at MR imaging: injury patterns of medial patellar softtissue restraints and osteochondral injuries of the inferomedial patella. Radiology 225:736-743

9. Hautamaa PV, Fithian DC, Kaufman KR, Daniel DM, Pohlmeyer AM (1998) Medial soft tissue restraints in lateral patellar instability and repair. Clin Orthop Relat Res 349:174-182

10. Mariani PP, Liguori L, Cerullo G, Iannella G, Floris L (2010) Arthroscopic patellar reinsertion of the MPFL in acute patellar dislocations. Knee Surg Sports Traumatol Arthrosc 19:628-633

11. Nomura E, Horiuchi Y, Inoue M (2002) Correlation of MR imaging findings and open exploration of medial patellofemoral ligament injuries in acute patellar dislocations. Knee 9:139-143

12. Sallay PI, Poggi J, Speer KP, Garrett WE (1996) Acute dislocation of the patella: a correlative pathoanatomic study. Am J Sports Med 24:52-60

13. Sanders TG, Morrison WB, Singleton BA, Miller MD, Cornum KG (2001) Medial patellofemoral ligament injury following acute transient dislocation of the patella: MR findings with surgical correlation in 14 patients. J Comput Assist Tomogr 25:957-962

14. Schöttle PB, Schmeling A, Rosenstiel N, Weiler A (2007) Radiographic landmarks for femoral tunnel placement in medial patellofemoral ligament reconstruction. Am J Sports Med 35:801-804

15. Servien E, Fritsch B, Lustig S, Demey G, Debarge R, Lapra C, Neyret P (2011) In vivo positioning analysis of medial patellofemoral ligament reconstruction. Am J Sports Med 39:134-139

16. Sillanpää PJ, Peltola E, Mattila VM, Kiuru M, Visuri T, Pihlajamäki H (2009) Femoral avulsion of the medial patellofemoral ligament after primary traumatic patellar dislocation predicts subsequent instability in men: a mean 7-year nonoperative follow-up study. Am J Sports Med 37:1513-1521

17. Stefancin JJ, Parker RD (2007) First-time traumatic patellar dislocation: a systematic review. Clin Orthop Relat Res 455:93-101

18. Warren LF, Marshall JL (1979) The supporting structures and layers on the medial side of the knee: an anatomical analysis. J Bone Joint Surg Am 61:56-62 